\title{
Avaliação das dificuldades de aprendizado em Bioquímica dos discentes da Universidade Federal do Piauí
}

\author{
Evaluation of learning difficulties in Biochemistry from the Federal University of Piauí students
}

\author{
Raíssa Silva Bacelar de Andrade ${ }^{1^{*}}$, Ayres Fran da Silva e Silva ${ }^{2}$, Maximiliano de Souza Zierer ${ }^{3}$ \\ ${ }^{1}$ Universidade Federal do Piauí - UFPI - Teresina/PI, Brasil. \\ ${ }^{2}$ Rede Nordestina de Biotecnologia - RENORBIO - Universidade Federal do Piauí - UFPI - Teresina/PI, \\ Universidade Federal Rural da Amazônia - UFRA, Campus Parauapebas, Brasil. \\ ${ }^{3}$ Departamento de Bioquímica e Farmacologia - Centro de Ciências da Saúde, Universidade Federal do Piauí - \\ UFPI, Brasil. \\ *e-mail: raissa_sba@hotmail.com
}

\begin{abstract}
Biochemistry is a basic subject present in the curriculum of the various health and natural sciences courses. However, the literature indicates a great difficulty for undergraduates facing the learning of this subject. This study aimed to evaluate, through a questionnaire, the difficulty degree of learning in Biochemistry presented by the students, in order to stimulate discussions about teaching-learning strategies to try to minimize the problem. We found that $86,5 \%$ of the students evaluated presented a difficulty degree of learning in Biochemistry from medium to high. In addition, $62.8 \%$ of the students reported had learning difficulties in other subjects in their undergraduate courses. Associated with these data, $81.03 \%$ of the students recognized that they had learning difficulties in high school, with the most mentioned subjects being mathematics (44.73\%) and chemistry (38.98\%). As a consequence of this problem, $21.4 \%$ of the total number of students evaluated was repeaters in the subject of Biochemistry, which represents a significant group of students with great learning difficulties. We verified that the difficulties of learning in Biochemistry have multiple causes, and probably the most important is the deficiency in the training of students coming from elementary and middle schools.
\end{abstract}

Keywords: learning difficulties; biochemistry; students.

\section{Resumo}

A Bioquímica é uma das disciplinas básicas mais presentes na grade curricular dos diversos cursos da área da saúde e das ciências da natureza. Contudo, a literatura aponta para uma grande dificuldade dos alunos de graduação frente ao aprendizado desta disciplina. Esse trabalho teve como objetivo avaliar, por meio de um questionário, o grau de dificuldade de aprendizado em Bioquímica apresentado pelos discentes, a fim de levantar discussões sobre estratégias de ensino-aprendizagem para tentar minimizar o problema. Constatamos que $86,5 \%$ dos alunos pesquisados apresentaram um grau de dificuldade de aprendizado em Bioquímica de médio a alto. Além disso, $62,8 \%$ dos alunos relataram ter dificuldades de aprendizado em outras disciplinas em seus cursos de graduação. Associado a esses dados, $81,03 \%$ dos estudantes reconheceram ter passado por dificuldades de aprendizado no ensino médio, sendo que as disciplinas mais mencionadas foram matemática $(44,73 \%)$ e química $(38,98 \%)$. Como consequência desse problema, $21,4 \%$ do total dos discentes pesquisados eram repetentes na disciplina de Bioquímica, o que representa um grupo significativo de alunos com grandes dificuldades de aprendizado. Verificamos que as dificuldades de aprendizado em Bioquímica possuem múltiplas causas, sendo que provavelmente a mais importante é a deficiência na formação dos alunos vindos dos ensinos fundamental e médio.

Palavras-chave: dificuldades de aprendizado; bioquímica; estudantes. 


\section{Introdução}

A Bioquímica é uma disciplina do núcleo básico da área da saúde. Ela permite investigar e compreender o ser humano, auxiliando na busca de soluções para os problemas de saúde. A aplicação correta dos conhecimentos em Bioquímica é fundamental para atuação de bons profissionais [1]. Além disso, a disciplina está presente em diferentes cursos da graduação, a qual aborda conceitos relacionados a micro e macromoléculas e reações químicas que ocorrem em nosso organismo. Apesar dos esforços para que a Bioquímica seja apresentada de forma coerente e organizada, ela é definida pelos estudantes como uma disciplina complexa, apresentando uma coleção de estruturas químicas e difícil de ser assimilada [2].

Nos últimos anos, observou-se ainda em grande parte das instituições de ensino superior do Brasil um certo grau de dificuldade no aprendizado de Bioquímica por parte dos alunos ingressantes em cursos de saúde, sendo esta uma disciplina básica para a grade curricular de cada um desses cursos, assim como física, cálculo e química em outros cursos, que também atingem altos níveis de reprovação. Além disso, os alunos de Bioquímica a definem como uma coleção de estruturas químicas e reações, com dificuldade de assimilação e desintegrada de sua prática profissional [3]. Na estrutura instrumental dos currículos vigentes, o reconhecimento da importância das disciplinas básicas ocorre tardiamente, quando os conceitos que exploram são necessários para o exercício profissional [4].

A este mesmo passo, a educação superior passa por uma mudança de paradigma em relação ao ensino e à aprendizagem; de um ensino centrado no professor para processos de aprendizagem focados nos estudantes, caracterizando as chamadas metodologias ativas de ensino-aprendizagem [5].

Na Universidade Federal do Piauí, por exemplo, a Bioquímica está presente na grade curricular de diversos cursos que possuem disciplinas ministradas no Centro de Ciências da Saúde, a saber: Odontologia, Medicina, Educação Física, Biologia, Química, Nutrição, Medicina Veterinária, Farmácia, Enfermagem e Agronomia. Dentre estes cursos, há os mais variados níveis de reprovação durante cada semestre. A partir dessa constatação, o presente trabalho propõe-se a investigar junto aos discentes quais são os motivos das suas dificuldades de aprendizado em Bioquímica, visando propiciar reflexões sobre as possíveis estratégias de ensino-aprendizado que permitam minimizar o problema. 


\section{Metodologia}

\subsection{População-alvo e universo do estudo}

A população do estudo foi composta por alunos matriculados na disciplina de Bioquímica do Departamento de Bioquímica e Farmacologia da Universidade Federal do Piauí entre os anos de 2014 e 2015, nos cursos de graduação de Odontologia, Medicina, Educação Física, Biologia, Química, Nutrição e Medicina Veterinária.

\subsection{Amostragem}

A amostra foi calculada com nível de confiança de $95 \%$ e um erro amostral de 5\%. Para os semestres de 2014.2, 2015.1 e 2015.2, foi obtida uma amostragem total de 622 alunos. Destes, 64 alunos cursavam Medicina, 68 alunos cursavam Educação Física, 73 alunos cursavam Ciências Biológicas, 90 alunos cursavam Medicina Veterinária, 97 alunos cursavam Química, 106 alunos cursavam Odontologia e 124 alunos cursavam Nutrição.

O número médio de alunos por turma foi de 23,71 alunos/professor. Por curso, o número médio de alunos por turma foi: Medicina, 20 alunos/professor; Educação Física, 25 alunos/professor; Biologia, 28 alunos/professor; Medicina Veterinária, 28 alunos/professor; Química, 20 alunos/professor; Odontologia 20 alunos/professor e Nutrição, 25 alunos/professor. Os estudantes foram escolhidos aleatoriamente entre os que aceitaram espontaneamente participar da pesquisa e estavam no final do semestre (última semana de aula) quando cursavam a disciplina. Os questionários foram respondidos anonimamente.

\subsection{Instrumento da coleta de dados}

As informações sobre as dificuldades de aprendizado em Bioquímica e os dados pessoais (sexo, idade, grau de instrução dos pais) foram obtidos através de um questionário que contém 33 questões objetivas e 2 questões subjetivas, bem como as perguntas relacionadas ao foco da pesquisa. Os questionários foram aplicados durante três semestres consecutivos. 


\subsection{Análise dos dados}

Os dados foram processados pelo programa SPSS Statistics para Windows ${ }^{\circledR}$ versão 10.0 (SPSS Inc., 2000). Na análise estatística foram utilizados a média, o desvio padrão e o intervalo de confiança.

\subsection{Aspectos éticos}

A inclusão dos discentes no estudo foi realizada mediante a assinatura do termo de consentimento livre e esclarecido, de acordo com as diretrizes da resolução 196/96 do Conselho Nacional de Saúde. O protocolo da pesquisa foi submetido ao Comitê de Ética em Pesquisa da Universidade Federal do Piauí antes de iniciar a pesquisa, e foi aprovado com o CAAE 02340414.4.0000.5214.

\section{Resultados e Discussão}

No depto. de Bioquímica e Farmacologia da UFPI, a disciplina de Bioquímica é ofertada no primeiro período de cada curso de graduação, exceto para os cursos de Química e de Biologia, onde é ofertada no quarto período. A carga horária é de 90h/aula por semestre nos cursos de Odontologia, Medicina, Educação Física, Nutrição e Medicina Veterinária, e de 60h/aula por semestre nos cursos de Química e Biologia. Apesar da diferença de carga horária, as aulas e os conteúdos das disciplinas de Bioquímica dos diferentes cursos de graduação são equivalentes em aproximadamente $70 \%$.

Nos cursos onde a disciplina de Bioquímica possui uma maior carga horária, são ministrados alguns conteúdos teóricos e práticos a mais. Com exceção dos cursos de Química e de Biologia, os discentes dos demais cursos de graduação não tem nenhuma disciplina prévia envolvendo conhecimentos de Química geral antes de cursar a disciplina de Bioquímica.

A nossa amostra foi constituída de 622 alunos, sendo $62,7 \%$ do sexo feminino e $37,3 \%$ do sexo masculino, com uma idade média de 20,98 anos e desvio padrão de $\pm 3,64$. Desse total de alunos, a maioria $(58,4 \%)$ cursou o ensino médio em escolas da rede privada, enquanto $37,3 \%$ eram provenientes de escolas públicas e apenas 4,2\% havia cursado o ensino médio em ambas as redes de ensino. Esse dado contraria a última pesquisa do INEP (Instituto Nacional de Estudos e Pesquisas), que aponta para uma maior porcentagem de alunos oriundos de escolas públicas matriculados em universidades públicas federais (Censo Superior - INEP 2014) [6]. 
Para analisar de uma forma geral o grau de dificuldade de aprendizado dos alunos na disciplina de Bioquímica, decidimos agrupá-los em quatro graus: nenhum, baixo, médio e alto. Para isso, os alunos atribuíram uma nota de 0 a 10 quanto ao seu grau de dificuldade de aprendizado em Bioquímica, sendo zero correspondente a nenhuma dificuldade; entre 1 a 3, dificuldade baixa; de 4 a 6, dificuldade mediana; e, de 7 a 10, alta dificuldade de aprendizado. (Tabela 01).

Tabela 01. Distribuição dos alunos segundo o grau de dificuldade de aprendizado na disciplina de

\begin{tabular}{c|cc} 
& Bioquímica. & \% \\
\hline Grau de dificuldade & $\boldsymbol{n}$ & 1,3 \\
\hline Nenhum & 8 & 11,7 \\
Baixo & 73 & 44,5 \\
Médio & 277 & 42,0 \\
Alto & 261 & 0,5 \\
Não respondeu & 3 & $\mathbf{1 0 0}$ \\
Total & $\mathbf{6 2 2}$ &
\end{tabular}

Constatamos que a maioria dos discentes encontrava-se com um grau de dificuldade média $(44,5 \%)$ ou alta $(42 \%)$ no aprendizado da disciplina de Bioquímica, corroborando com a literatura $[7,8]$. Tendo em vista essa situação, inúmeros autores propõem mudanças de paradigmas no ensino da disciplina, discutindo-se inclusive a ideia de não mais organizar uma disciplina de Bioquímica em tópicos clássicos, mas em determinar previamente as habilidades a serem dominadas pelos alunos no término de um curso básico, as quais podem incluir a aplicação de determinados conteúdos à atividade prática, bem como a orientação na leitura de trabalhos acadêmicos, permitindo a atualização de conhecimentos na velocidade em que as informações são divulgadas [9].

É importante ainda ressaltar que os primeiros anos da graduação, onde geralmente está presente a disciplina Bioquímica, são considerados um período crítico, pois exige adaptação e integração ao novo ambiente, logo muitos alunos revelam dificuldades pessoais e acadêmicas, havendo inclusive um aumento nos níveis de psicopatologia da população universitária [10].

Quando analisamos as dificuldades de aprendizado em Bioquímica para cada um dos cursos de graduação pesquisados, verificamos que o maior nível de dificuldade se encontrava entre os alunos de Educação Física (com uma média de dificuldade de aprendizado de 6,7), seguido pelos de Nutrição (média de 6,5), Biologia $(6,3)$ e Medicina Veterinária $(6,23)$.

Os cursos nos quais os alunos apresentaram menor grau de dificuldade de aprendizado em Bioquímica foram Odontologia (média de dificuldade de 5,44), Medicina (média de dificuldade de 5,73) e Química (média de dificuldade de 5,80) (Figura 01). 




Figura 01. Média do grau de dificuldade em Bioquímica de acordo com o curso de graduação.

Em relação às dificuldades dos alunos por tópico ministrado dentro da disciplina de Bioquímica, pedimos aos discentes que escolhessem três entre os temas ministrados que eles considerassem os mais difíceis. Verificamos que o maior índices de dificuldade de aprendizado foram encontrados nos assuntos de enzimas (32,5\% dos alunos), seguido por biosinalização $(33,3 \%)$ e a integração do metabolismo $(27,5 \%)$ (Figura 02)

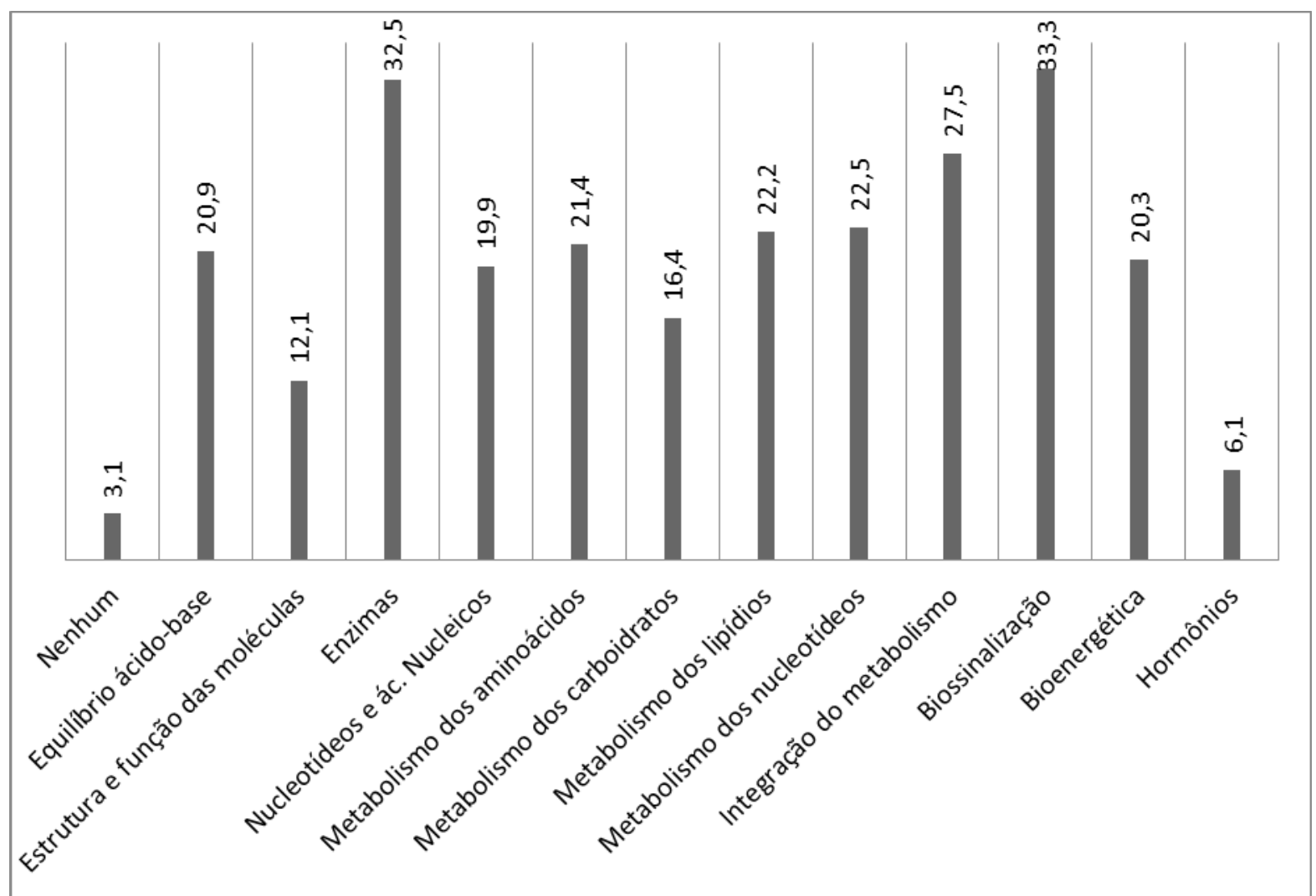

Figura 02. Porcentagem de alunos com dificuldades de aprendizado por assunto em Bioquímica. 
$\mathrm{Na}$ percepção dos discentes, estes são os temas mais intrincados e de difícil assimilação, e, portanto, requerem por parte dos docentes um maior cuidado na condução do processo de ensino-aprendizagem.

Para estes temas, sugerimos a utilização de diferentes estratégias de ensinoaprendizagem, como a realização de exercícios em sala de aula, o uso de animações em vídeo e atividades lúdicas e interativas, como a construção de modelos e maquetes em sala de aula $[5,9]$.

Para avaliar os possíveis motivos para o elevado grau de dificuldade de aprendizado na disciplina de Bioquímica, sugerimos aos discentes que escolhessem esses motivos dentro de uma relação predeterminada, obtida em nosso estudo piloto. Assim, no atual estudo, cada discente pôde eleger três motivos para justificar as suas dificuldades de aprendizado em Bioquímica. Como resultado, obtivemos que os principais motivos citados pelos alunos foram o excesso de conceitos envolvidos no estudo da Bioquímica (54\%), seguido do fato deles considerarem o volume de informação muito grande a ser abordado em pouco tempo (48,2\%) e a presença de outras disciplinas complexas na grade curricular de cada curso (39,4\%) (Figura 03).

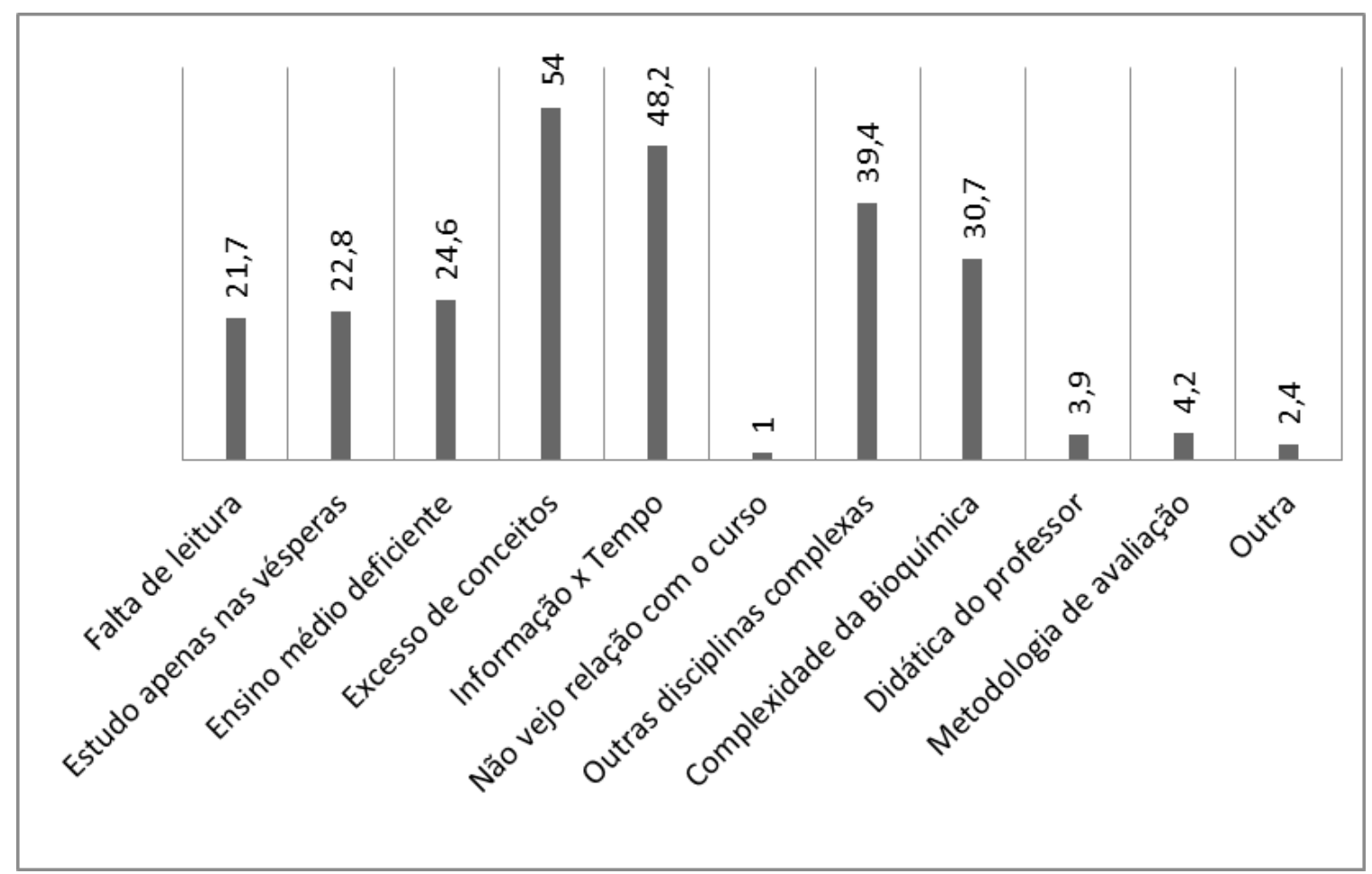

Figura 03. Porcentagem de alunos por motivo alegado para as dificuldades de aprendizado em Bioquímica.

Outros motivos citados foram a complexidade da disciplina (30\%), o ensino médio deficiente (24,6\%), o estudo para as avaliações somente nas vésperas das mesmas $(22,8 \%)$ e a falta de leitura $(21,7 \%)$. Cabe ressaltar que, dentre os motivos menos citados 
pelos discentes para justificar as suas dificuldades de aprendizado em Bioquímica, tivemos as metodologias de avaliação $(4,2 \%)$, a didática do professor $(3,9 \%)$ e não ver a relação da disciplina de Bioquímica com o próprio curso de graduação (1\%), além de outros motivos não especificados (2,4\%). Em relação as metodologias de avaliação, os professores do Departamento de Bioquímica e Farmacologia da UFPI utilizam uma ampla variedade de ferramentas de avaliação: provas, seminários, relatórios, estudos dirigidos, minitestes e construção de modelos didáticos em sala de aula. Deste modo, verificamos que, na percepção dos estudantes, há um baixo índice de desaprovação quanto às metodologias empregadas para avaliar a aprendizagem em Bioquímica. Decerto que a Bioquímica é uma disciplina complexa, mas essa complexidade pode ser superada desde que os alunos tenham uma formação básica adequada, possuam gosto pela leitura, curiosidade, disciplina e motivação para estudar. Considerando que os alunos citaram como um dos motivos para as suas dificuldades em aprendizado em Bioquímica o ensino médio deficiente $(24,6 \%)$, consideramos investigar a formação prévia dos alunos.

Apresenta-se na Figura 4 o percentual de estudantes que afirmaram ter tido dificuldades de aprendizado no ensino médio. Constatamos que $81,03 \%$ dos estudantes ( $n=504)$ relataram dificuldades de aprendizado, um índice muito elevado, enquanto somente $18,97 \%$ dos estudantes $(n=118)$ não relataram ter dificuldades de aprendizagem no ensino médio.

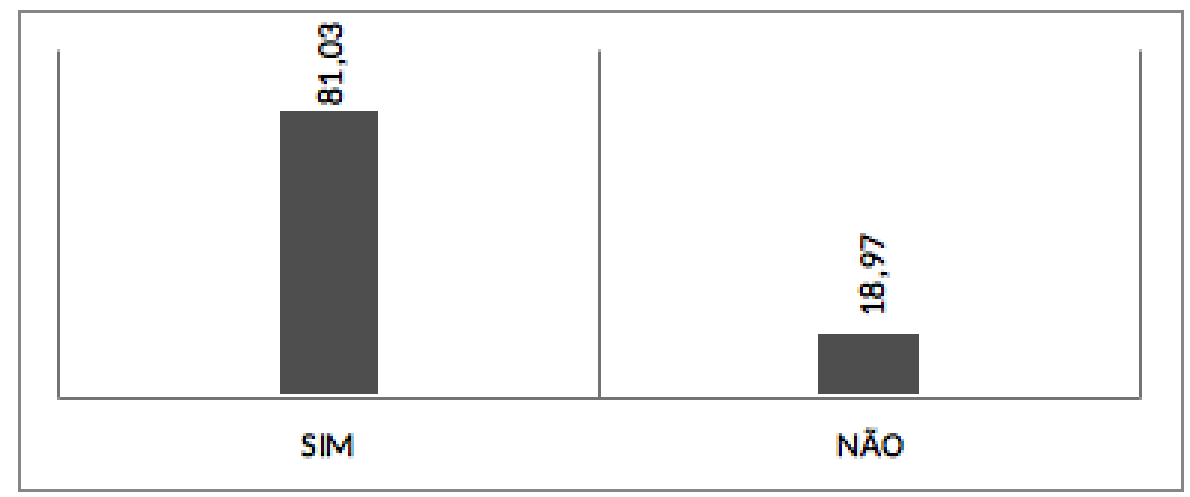

Figura 04. Porcentagem de alunos que apresentaram dificuldades de aprendizado no ensino médio.

Nossos resultados são compatíveis com os dados divulgados no relatório de 2015 do PISA (Programa Internacional de Avaliação de Estudantes), realizado pela OCDE (Organização para Cooperação e Desenvolvimento Econômico), que avaliou 23.141 estudantes brasileiros de 841 escolas, nas idades de 15 e 16 anos. O relatório mostrou que o desempenho dos alunos no Brasil está muito abaixo da média dos alunos de países da OCDE em ciências (obtivemos 401 pontos, comparados à média de 493 pontos dos 
países da OCDE), em leitura (407 pontos, comparados à média de 493 pontos) e em matemática (377 pontos, comparados à média de 490 pontos) [11]. O Brasil também vem caindo no ranking mundial: dentre 70 países avaliados, o país ficou na $63^{a}$ posição em ciências, na $59^{a}$ em leitura e na $66^{a}$ colocação em matemática. A partir destas constatações, não se pode esperar um bom desempenho em uma disciplina complexa como a Bioquímica quando os alunos ingressam no nível superior com deficiências em ciências e em conceitos científicos em geral. Para piorar, muitos alunos sequer dominam a leitura e/ou possuem aversão a mesma, sendo que esse padrão parece não sofrer modificação desde 2006, segundo o PISA. Ou seja, o ensino no Brasil está atualmente estagnado e encontra-se entre os piores do mundo. [11].

Ainda quanto às dificuldades em ciências no ensino médio brasileiro, a OCDE mostra que, em um quadro comparativo dos 27 estados da federação, o Piauí aparece na vigésima posição, o que é ainda mais grave. Os três melhores Estados do Brasil são o Espírito Santo, o Distrito Federal e o Paraná (notas médias de 435, 426 e 425 pontos, respectivamente). No Nordeste, o Piauí (com nota média de 380 pontos) está à frente da Alagoas, Bahia, Rio Grande do Norte, Maranhão, Sergipe e Paraíba, porém ficou atrás do Ceará (o melhor do Nordeste, com nota média de 401, o único da região acima de 400 pontos) e Pernambuco (nota média de 383 pontos).

Segundo as Sinopses Estatísticas dos Censos da Educação Superior do MEC de 2014 [6], o número de alunos matriculados (em cursos presenciais e à distância) por ano em Instituições de Ensino Superior (IES) excede em muito o número de concludentes. Os dados do referido ano constam como 7.828.013 alunos matriculados em IES públicas e privadas, contra apenas 1.027.092 concluintes. Esta relação leva à conclusão de que o grau de evasão universitária é alto. Na Universidade Federal do Piauí, na sinopse estatística do mesmo ano, o número de concluintes em cursos presenciais foi de 3.146, contra 22.488 matrículas. É provável que esse alto grau de evasão esteja relacionado às dificuldades de aprendizado enfrentadas durante a graduação, as quais tem profundas raízes no desempenho deficiente dos ensinos fundamental e médio.

Também examinamos quais eram as disciplinas do ensino médio onde os alunos relataram possuir as maiores dificuldades de aprendizado (Figura 05). Matemática $(44,73 \%)$ e Química (38,98\%) foram as duas disciplinas mais citadas, sendo clara a correlação entre as dificuldades em Química no ensino médio e as dificuldades em aprendizado em Bioquímica no ensino superior. Além disso, os alunos relataram dificuldades em Outras disciplinas (12,23\%) - grupo que incluiu o somatório de 
Português, História, Inglês e Geografia, além de Física $(11,02 \%)$ e, por último, Biologia, com apenas 6,23\% (Figura 05). Segundo o relatório PISA, em matemática, 70,3\% dos alunos brasileiros ficaram abaixo do nível 2 - que é o patamar mínimo na OCDE considerado como necessário para que o estudante exerça plenamente a sua cidadania. [11]. Os nossos estudantes não conseguem responder às questões da disciplina de matemática com clareza e nem identificar ou executar procedimentos rotineiros de acordo com instruções diretas. [11]. Quando se avaliaram as habilidades de investigação científica, o Brasil chegou a 401 pontos, bem abaixo da média da OCDE (493 pontos) e em queda desde 2012.

Observou-se que $56,5 \%$ dos nossos estudantes tiveram um desempenho abaixo do nível 2, ou seja, não são capazes de identificar uma explicação científica, interpretar dados e identificar a questão abordada em um projeto experimental simples de complexidade mediana [13]. Quanto à leitura, metade dos alunos brasileiros avaliados continua com dificuldades de interpretação, sendo que $51 \%$ dos estudantes não possuem o nível que a OCED estabelece como necessário para que se possa exercer plenamente a cidadania (não ultrapassam o nível 2), tendo dificuldades de trabalhar textos e documentos oficiais, tais como notas públicas e notícias, e ainda possuem problemas em interpretar informações e integrar textos.

O ensino e a aprendizagem da disciplina de Bioquímica é desafiador pela própria natureza desse conhecimento. Por exemplo, a Bioquímica possui uma terminologia ampla e específica (nomes, fórmulas e estruturas químicas), que exige um alto grau de abstração e um conhecimento básico de Química [13]. A Bioquímica é uma área complexa, da qual os alunos trazem, em geral, uma série de conceitos incompletos ou equivocados, o que pode explicar as altas taxas de dificuldade de aprendizado na disciplina. Não nos surpreende que estudantes com deficiências em ciências, química e em leitura e interpretação de textos tenham grande dificuldade de aprendizado em Bioquímica. Segundo Ausubel [14], é ainda de fundamental importância entender que o processo de aprendizagem depende do conhecimento que o estudante já possui, ou seja, de seu conhecimento prévio.

Assim, compreende-se como uma real aprendizagem, uma mudança no conhecimento já existente, tanto do ponto de vista quantitativo e, de grande importância, qualitativo - mudança na qualidade do conhecimento já existente em função do novo conhecimento, quando aprendizagem significativa pode ser vista como um processo de Mudança Conceitual [8]. 




Figura 05. Porcentagem de alunos com dificuldades de aprendizado no ensino médio por disciplina.

Outro dado significativo que obtivemos é que, embora a maioria dos alunos (78,6\%) estivesse cursando a disciplina de Bioquímica pela primeira vez, um contingente de $18,3 \%$ cursava Bioquímica pela segunda vez, enquanto 2,9\% cursava pela terceira vez. (Tabela 02). Assim, 21,4\% do total dos discentes pesquisados já eram repetentes na disciplina de Bioquímica, o que representa um expressivo grupo de alunos com grandes dificuldades de aprendizado, possuindo um histórico de reprovação na disciplina.

Este número elevado de repetentes provavelmente é uma consequência das deficiências de formação dos estudantes que chegam do ensino médio e ingressam na universidade, e mais tarde se manifestará nas elevadas taxas de evasão observadas na nossa universidade. [6].

Tabela 02. Frequência e porcentagem de alunos quanto ao número de vezes em que estavam cursando a disciplina de Bioquímica.

\begin{tabular}{ccc}
\hline $\begin{array}{c}\text { Quantidade de vezes que a } \\
\text { disciplina foi cursada }\end{array}$ & $\boldsymbol{n}$ & $\%$ \\
\hline $1^{a}$ vez & 489 & 78,6 \\
$2^{a}$ vez & 114 & 18,3 \\
$3^{a}$ vez & 18 & 2,9 \\
Mais de três vezes & 1 & 0,2 \\
Total & $\mathbf{6 2 2}$ & $\mathbf{1 0 0}$ \\
\hline
\end{tabular}

Outro aspecto relevante que levantamos é que, considerando a grade curricular de cada curso avaliado, 62,8\% dos alunos relataram também possuir dificuldades de aprendizado em outras disciplinas específicas do seu curso de graduação (Figura 06). 


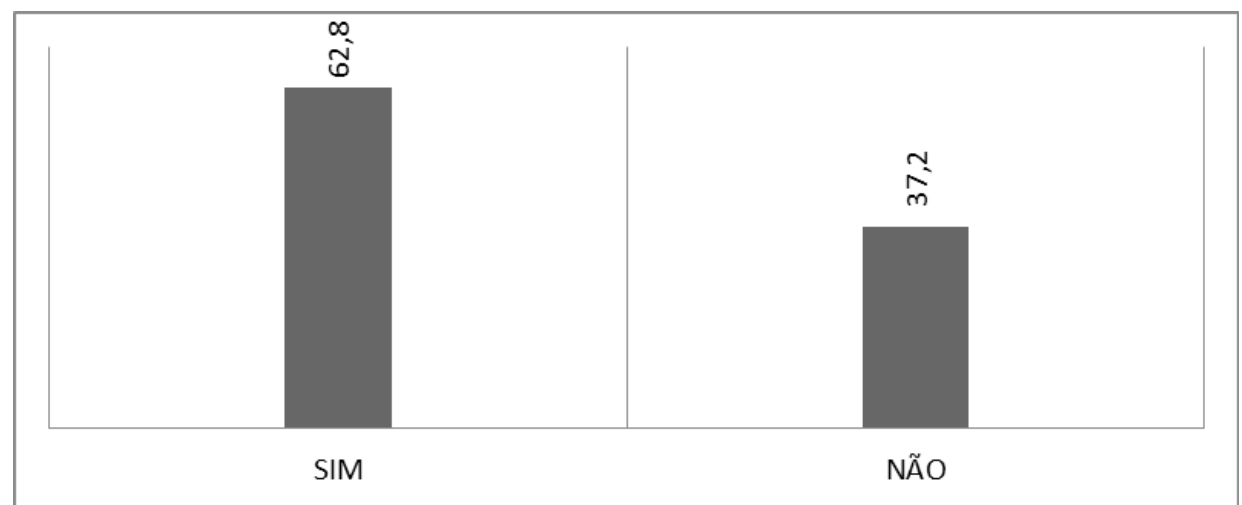

Figura 06. Porcentagem de alunos quanto a dificuldades de aprendizado em outras disciplinas da graduação.

Também investigamos qual era a frequência com que os discentes estudavam Bioquímica fora da sala de aula. Verificamos que, embora $54,1 \%$ estudassem dois ou mais dias da semana, por outro lado encontramos 20,9\% que afirmaram só estudar na véspera das avaliações, enquanto outros $25 \%$ só estudavam uma vez por semana, o que em ambos os casos consideramos insuficiente para o aprendizado de uma disciplina como a Bioquímica (Tabela 03).

Portanto, quase $46 \%$ dos alunos de Bioquímica possuem uma rotina de estudos inadequada: muitas vezes protelam os estudos, deixam acumular vários conteúdos ministrados, e quando entram em contato com assuntos que requerem muito mais do que uma dedicação superficial - voltada somente para o decoreba de nomes e conceitos - acabam tendo grandes dificuldades de aprendizagem. Segundo Oliveira e Oliveira (2007), os estudantes universitários ingressantes apresentam, no Brasil, um baixo desempenho na compreensão em leitura, considerando o esperado para esse nível de escolaridade, logo, podemos inferir que esta dificuldade pode estar associada à frequência do estudo, tendo em vista que a disciplina Bioquímica tem um vasto conteúdo para leitura em livros como parte do plano de ensino [12].

Tabela 03. Alunos distribuídos pela frequência semanal de estudo em Bioquímica.

\begin{tabular}{ccc}
\hline Frequência com que estuda & $\boldsymbol{n}$ & $\%$ \\
\hline Um dia por semana & 149 & 25,0 \\
Dois dias por semana & 199 & 33,3 \\
Três dias por semana & 104 & 17,4 \\
Quatro ou mais dias por semana & 20 & 3,4 \\
Estuda apenas na véspera & 125 & 20,9 \\
Total & $\mathbf{6 2 2}$ & $\mathbf{1 0 0 , 0}$ \\
\hline
\end{tabular}

Pensando em superar esses vários obstáculos e aprimorar o processo de ensinoaprendizagem, muitos professores no nosso departamento recorrem a estudos dirigidos (exercícios) em sala de aula (Tabela 04). Segundo a opinião dos alunos, este é um recurso válido, visto que $50 \%$ deles relatou que os estudos dirigidos sempre os auxiliam, 
enquanto outros $31,8 \%$ consideraram que os exercícios em sala de aula frequentemente são proveitosos, ou seja, essa metodologia obteve uma aprovação de 81,8\%. De fato, a literatura pertinente afirma que o estudo dirigido coloca o aluno como centro de todas as atividades, descobrindo o prazer para aprender e sendo sujeito de suas ações, como resultado do processo ensino-aprendizagem [15].

A literatura mostra ainda a importância do uso de metodologias ativas em sala de aula como facilitadoras do aprendizado de Bioquímica, como, por exemplo, a técnica do "aprender-fazendo", na qual os alunos se tornam sujeitos do ensino, por meio de jogos, montagem de modelos estruturais de moléculas ou mesmo gincanas e dramatizações $[16,17]$.

Segundo Bartalo e Guimarães (2008), essas metodologias didáticas são inovadores, tendo em vista que, de modo geral, os estudantes exercem uma postura passiva, diante da aprendizagem, esperando uma postura ativa por parte do professor, apresentando argumentos convincentes para que os alunos estudem determinados conteúdos, organizando aulas interessantes e, quem sabe, divertidas. Barbosa et al. (2009) relatam que, no Brasil, pouca atenção é dada para a formação didática dos professores para a preparação de aulas lúdicas, com metodologias didáticas inovadoras [19].

Tabela 04. Quantidade e frequência dos alunos em relação a percepção do auxílio dos exercícios na aprendizagem em Bioquímica.

\begin{tabular}{ccc}
\hline Frequência & $\boldsymbol{n}$ & $\%$ \\
\hline Sempre & 311 & 50 \\
Frequentemente & 198 & 31,8 \\
Ocasionalmente & 81 & 13 \\
Raramente & 26 & 4,2 \\
Nunca & 6 & 1 \\
Total & $\mathbf{6 2 2}$ & $\mathbf{1 0 0 , 0}$ \\
\hline
\end{tabular}

Finalmente, confirmamos que, na percepção dos discentes, as dificuldades em aprendizado na disciplina de Bioquímica não parecem estar relacionadas a uma didática deficiente dos docentes, visto que $91,6 \%$ a considerou boa ou excelente (Tabela 05), ou seja, houve um alto índice de aprovação quanto à didática dos professores. Isso confirma o dado mostrado na Figura 02 , onde apenas 3,9\% dos estudantes citaram a didática dos professores como um dos motivos para as suas dificuldades de aprendizado na disciplina.

De fato, diversos estudos colocam a figura do professor como facilitadora do processo ensino-aprendizagem, pois o profissional é capaz de criar diferentes abordagens sobre o conteúdo, permitindo aos alunos construir as suas diferentes concepções sobre os assuntos [20, 21, 22]. 
Segundo Veras e Ferreira (2010), a atuação dos professores é capaz de despertar o interesse no aprendizado dos alunos, fazendo com que estes busquem dar um retorno aos professores através do cumprimento das atividades solicitadas e da participação ativa nas aulas [23].

Tabela 05. Avaliação dos alunos quanto à didática dos professores de Bioquímica.

\begin{tabular}{ccc}
\hline Avaliação & $\boldsymbol{n}$ & $\%$ \\
\hline Excelente & 214 & 34,4 \\
Boa & 356 & 57,2 \\
Mediana & 45 & 7,2 \\
Ruim & 4 & 0,6 \\
Péssima & 3 & 0,5 \\
Total & $\mathbf{6 2 2}$ & $\mathbf{1 0 0 , 0}$ \\
\hline
\end{tabular}

\section{Considerações finais}

As dificuldades de aprendizado em Bioquímica possuem múltiplas causas, sendo que provavelmente a mais importante é a deficiência na formação dos alunos vindos do ensino fundamental e médio. A maioria dos alunos relatou possuir dificuldades de aprendizado no ensino médio, fator que, como dificuldade cognitiva geral, vem interferindo no aprendizado de disciplinas do ensino superior, como a Bioquímica.

Dentro de matérias específicas do ensino médio, o maior nível de dificuldade foi em Matemática e Química, sendo que esta última é específica para os cursos da área da saúde e uma das bases da Bioquímica, fato que certamente contribui para a posterior dificuldade nesta disciplina. Os nossos dados corroboram o relatório Pisa de 2015 da OCDE em relação a atual situação educacional brasileira, que está mal colocada nos rankings mundiais e com um alto grau de evasão universitária apresentado no país.

Para tentar minimizar as dificuldades de aprendizado em Bioquímica, sugerimos atividades que facilitem o processo de ensino-aprendizado, como, por exemplo, estudos dirigidos (exercícios) em sala de aula, que os nossos alunos consideraram uma alternativa que sempre ajuda e facilita a aquisição do conhecimento.

Além disso, é essencial sair da mesmice e empregar metodologias pedagógicas que permitam aos estudantes universitários a livre expressão da sua criatividade, proporcionando uma aprendizagem mais ativa e participativa, como a construção de modelos e maquetes e/ou a dramatização de processos bioquímicos em sala de aula. Contudo, mesmo com a utilização dessas estratégias, não será possível obter grandes avanços na educação superior brasileira como um todo sem uma mudança radical de qualidade nos nossos ensinos fundamental e médio, que estão estagnados em patamares muito baixos 


\section{Referências}

[1] Santos VT, Anacleto C. Monitorias como ferramenta auxiliar para aprendizagem da disciplina bioquímica: uma análise no Unileste-MG. Rev Ensino Bioquim. 2007; 5(1): E-E8.

[2] Pinheiro TDL, Silva JAD, Souza PRMD, Nascimento MMD, Oliveira HDD. Ensino de Bioquímica para acadêmicos de Fisioterapia: visão e avaliação do discente. Rev Ensino Bioquim. 2009; 7(1): 25-35.

[3] Vargas LMA. Bioquímica e a aprendizagem baseada em problemas. Rev Ensino Bioquim. 2001;1:1-5.

[4] Scatigno AC. Ensino de bioquímica no curso de nutrição em uma instituição de ensino superior da rede particular: diagnósticos e intervenções. Tese de doutorado - Universidade de São Paulo. 2010.

[5] Garzón JCV, Magrini ML, Costa C, Galembeck E. Realidade aumentada no ensino de vias metabólicas. Rev Ensino Bioquim. 2014;12(2):19-143.

[6] Instituto Nacional de Estudos e Pesquisas Educacionais Anísio Teixeira (INEP). Sinopses Estatísticas da Educação Superior 2015. Disponível em: http://portal.inep.gov.br/superior-censosuperior-sinopse

[7] Beckhauser PF, Almeida EM, Zeni ALB. O universo discente e o ensino de bioquímica. 2006;2:B1-B7.

[8] Zeni ALB. Conhecimento prévio para a disciplina de bioquímica em cursos da área da saúde da Universidade Regional de Blumenau-SC. Rev Ensino Bioquim. 2010;2(1):B1-B14.

[9] Feldberg RS. The new biochemistry: In praise of alternate curricula. Bioch Mol Biol Education. 2001; (29):222-224.

[10] Cunha SM, Carrilho DM. O processo de adaptação ao ensino superior e o rendimento acadêmico. Psicol Esc Educ. 2005;9(2).

[11] OECD. Programme for International Student Assesment (PISA): Results from PISA 2015. Pisa 2015 Database. Disponível em: https://www.oecd.org/pisa/pisa-2015-Brazil-PRT.pdf

[12] Oliveira RAM, Oliveira KL. Leitura e condições de estudo em universitários ingressantes. Revista de Psicologia da Vetor Editora. 2007;8(1);51-59.

[13] Schönborn KJ, Anderson TR. Bridging the educational research-teaching practice gap: foundations for assessing and developing biochemistry students' visual literacy. Biochem Mol Biol Educ. 2010; 38(5): 34754.

[14] Ausubel DP, Novak JD, Hanesian H. (1978) Educational Psychology: A Cognitive view. Holt, Rinehardt\& Winston. 1978.

[15] Okane ESH. O estudo dirigido como estratégia de ensino na educação profissional em enfermagem. Dissertação de mestrado - Universidade de São Paulo. 2004.

[16] Mitre SM, Siqueira-Batista R, Mendonça JMG, Pinto NMM, Meirelles CAB, Pinto-Porto C et al. Metodologias ativas de ensino-aprendizagem na formação profissional em saúde: debates atuais. Cienc. Saúde Coletiva. 2008;13(2):2133-2144.

[17] Zierer MS, Assis RC. A construção de modelos como estratégia para um ensino mais criativo nas disciplinas de bioquímica e biologia molecular. Diálogos \& Ciência (Online), v. 8, p. 1-14, 2010.

[18] Bartalo L, Guimarães SER. Estratégias de estudo e aprendizagem de alunos universitários: um estudo exploratório. Inf Inf. 2008;13(2);1-14.

[19] Barbosa $\mathrm{N}$ et al. A expressividade do professor universitário como fator cognitivo no ensino-aprendizagem. Ciências \& Cognição. 2009;14(1);75-102.

[20] Mortimer EF. Pressupostos epistemológicos para uma metodologia de ensino de química: mudança conceitual e perfil epistemológico. Química Nova. 1992;15(3):242-249. 
[21] Silveira FT, Rocha JBT. Produção científica sobre estratégias didáticas utilizadas no ensino de Bioquímica: uma revisão sistemática. Journal of Biochemistry Education. 2016;14(1):7-21.

[22] Vilhena L, Mota FNN, Oliveira FC, Batista FTS, Drefs YDS, Rosa LO. Jogo Bozó Genético: uma proposta didática como alternativa para o ensino da replicação de DNA no Ensino Médio. Journal of Biochemistry Education. 2016;14(3);57-67.

[23] Veras RS, Ferreira SPA. A afetividade na relação professor-aluno e suas implicações na aprendizagem, em contexto universitário. Educar em Revista. 2010;38;219-235.

\section{Agradecimentos.}

Agradecemos aos professores do Departamento de Bioquímica e Farmacologia da UFPI que colaboraram com a aplicação dos questionários nas suas turmas: Kátia Bonfim Leite de Moura Sérvulo, Fernando Aécio de Amorim Carvalho, Salete Maria da Rocha Cipriano Brito, Regina Célia de Assis e Osmar de Oliveira Cardoso. 